\title{
Excessive lodine Induces Thyroid Follicular Epithelial Cells Apoptosis by Activating HIF-1a- mediated Hypoxia Pathway in Hashimoto Thyroiditis
}

lili zhang ( $\nabla$ zhanglili1103@163.com )

Capital Medical University Affiliated Beijing Friendship Hospital

Lin Liu

Capital Medical University Affiliated Beijing Friendship Hospital

Ping Wang

Capital Medical University Affiliated Beijing Friendship Hospital

Linxue Qian

Capital Medical University Affiliated Beijing Friendship Hospital

Original Article

Keywords: Hashimoto's thyroiditis, apoptosis, HIF-1a, thyroid follicular cell

Posted Date: February 12th, 2021

DOI: https://doi.org/10.21203/rs.3.rs-191524/v1

License: (c) (i) This work is licensed under a Creative Commons Attribution 4.0 International License.

Read Full License 
Excessive iodine induces thyroid follicular epithelial cells apoptosis by activating HIF-1 $\alpha$-mediated hypoxia pathway in Hashimoto thyroiditis

Lili Zhang ${ }^{1}$; Lin Liu ${ }^{2}$; Ping Wang ${ }^{2 *}$, Linxue Qian ${ }^{1 *}$

${ }^{1}$ Department of Ultrasound, Beijing Friendship Hospital, Capital Medical University, Beijing, China

${ }^{2}$ Department of Liver Research Center, Beijing Friendship Hospital, Capital Medical University, Beijing, China

\section{*Corresponding authors:}

Linxue Qian

Department of Ultrasound, Beijing Friendship Hospital, Capital Medical University, Beijing, China.

E-mail: qianlinxue2002@163.com

Ping Wang

Department of Liver Research Center, Beijing Friendship Hospital, Capital Medical University, Beijing, China

E-mail: wangping2009@ccmu.edu.cn 


\section{Abstract}

Purpose Hashimoto thyroiditis (HT) is considered the most common autoimmune thyroid disease. A growing body of evidence suggests that HT incidence correlates with excessive iodine intake. We should probe the effects of excessive iodine intake in HT development and its possible mechanism.

Methods The study recruited 20 patients: 10 with HT and 10 with nodular goiter. We detected the expression of an apoptosis-related protein caspase-3 by immunohistochemistry. In vitro study, we explored the proliferation and apoptosis status in thyroid follicular cells (TFCs) stimulated at different iodine concentrations by MTT and flow cytometry. Then we performed RNA sequence analysis of Nthy-ori3-1 cells treated for $48 \mathrm{~h}$ with different $\mathrm{KI}$ concentrations to probe the underlying mechanism. Finally, we used RT-PCR to verify its results.

\section{Results}

We identified apoptosis in thyroid tissue obtained from HT patients coincides with the increase of caspase-3 levels. In vitro study, iodine suppressed proliferation of TFCs and promoted TFCs apoptosis in a dose-dependent manner. RNA sequence results showed that high concentration of iodine activate the hypoxia-inducible factor-1 $\alpha$ (HIF-1 $\alpha$ )mediated hypoxia pathway coincide with the upregulation of N-myc downregulated gene-1 (NDRG1) expression.

Conclusions Our study confirmed that excessive iodine adsorption activates the HIF$1 \alpha$-mediated hypoxia pathway to promote apoptosis of TFCs, which may be an important risk factor contributing to HT development. 
Key words: Hashimoto's thyroiditis, apoptosis, HIF-1 $\alpha$, thyroid follicular cell 


\section{Introduction}

It is well-known that iodine is an essential micronutrient for thyroid hormone synthesis, which undoubtedly plays a pivotal role in thyroid disease development. Mounting evidence suggests that severe iodine deficiency causes goiter and hypothyroidism, while excess iodine intake causes autoimmune thyroid disorders, including Hashimoto thyroiditis (HT) and Graves disease $[1,2]$. The increasing incidence of HT after the implementation of universal salt iodization supports these observations [2, 3, 4]. Furthermore, patients are prone to develop hypothyroidism and papillary thyroid carcinoma when exposed to excessive iodine [5].

HT is one of the most common autoimmune thyroid diseases, accounting for $5 \%$ of the worldwide population [6]. The main features of HT are chronic lymphocytic infiltration, varying degrees of thyroid follicular cells (TFCs) atrophy, and elevated titers of thyroid autoantibodies, mainly to thyroid peroxidase antibody (TPOAb) and thyroglobulin antibody (TgAb). It is now well established that genetic susceptibility and environmental factors inducing autoimmune disorder, including the overactivation of CD4+ T cells and reduction of T regulatory cells, results in the development of HT $[7,8]$. However, some studies have found that excessive iodine induces TFCs apoptosis, necrosis, and pre-infiltration of lymphocytes [9]. Thus, indicating that TFCs apoptosis preceding immune activation may be vital to cause HT.

Various internal and external stimuli could activate TFCs apoptosis, including DNA damage, the release of proinflammatory cytokines, and the aberrant expression of proapoptotic proteins [8]. Furthermore, a high number of apoptosis-related proteins levels changes during the development of HT like tumor necrosis factor-related 
apoptosis-inducing ligand (TRAIL), Fas ligand, caspases, and B-cell lymphoma (Bcl2) $[10,11]$. A previous study demonstrates that excessive iodine promotes TFCs apoptosis by suppressing autophagy, together with high levels of caspase- 3 in HT disease. However, until now, the mechanism of excessive iodine-induced TFCs apoptosis during HT development is unclear.

According to recent research, hypoxia inducible factor-1 (HIF-1) is an important transcription factor that mediates cells adaptive response under hypoxia conditions. HIF-1 consists of HIF- $1 \alpha$ and HIF-1 $1 \beta$ subunits, and HIF- $1 \alpha$ is the active subunit involved in the regulation of metabolism, angiogenesis, cell proliferation, and apoptosis [12]. HIF-1 $\alpha$ could also regulate the transcription of many apoptosis-related genes such as survivin, Bcl-2, and caspase- $3[13,14]$. This is in agreement with a recent study concluding that HIF-1 $\alpha$ can trigger hypoxia-induced apoptosis of alveolar epithelial cells during pulmonary fibrosis development [13]. Thus far, no studies have clarified the relationship between iodine, HIF-1 $\alpha$, and apoptosis of thyroid follicular cells. Our study found that excessive iodine activated HIF-1 $\alpha$-mediated hypoxia pathway and upregulated the expression of N-myc downregulated gene-1(NDRG1), promoting TFCs apoptosis. These findings indicate that excessive iodine is a precipitant in HT development, and HIF-1 $\alpha$ may be a potential biomarker of thyrocyte injury in HT.

\section{Materials and Methods:}

\section{Cell line and samples}

Thyroid follicular epithelial cell line Nthy-ori3-1 (Fuheng Biotechnology Co, Ltd.) was 
cultured in RPMI 1640 medium (Gibco, USA) supplemented with 10\% fetal bovine serum (Gibco, USA) and $2 \mathrm{mM}$ L-glutamine (Biological Industries, Kibbutz Beit Haemek, Israel). The thyroid tissues used in this study were obtained from 10 patients with HT and 10 with nodular goiter. HT was diagnosed according to clinical evaluation and pathological criteria, as previously described [15]. Nodular goiter was diagnosed according to the corresponding clinical and laboratory tests. All samples were collected from the Beijing Friendship Hospital affiliated to Capital Medical University. This study was approved by the Ethics Committee of the Beijing Friendship Hospital affiliated to Capital Medical University.

\section{Cell treatments}

We achieved different concentrations of iodine stimuli by diluting potassium iodide (KI) with cell culture media to a final concentration of $20-80 \mathrm{mM}$. Nthy-ori-3 cells were treated with $20-80 \mathrm{mM} \mathrm{KI}$ for $48 \mathrm{~h}$. The control group only consisted of untreated Nthyori-3 cells.

\section{Immunohistochemistry Staining}

Samples were fixed in $10 \%$ neutralized formalin, embedded in paraffin, and sectioned with a $4-5 \mu \mathrm{m}$ thickness. After deparaffinization and rehydration, antigen retrieval was performed by boiling the samples in EGTA Antigen Retrieval solution ( $\mathrm{pH}$ 8.0) and then washing the sections with phosphate-buffered saline (PBS). Thyroid sections were incubated with rabbit anti-human HIF-1 $\alpha$ (Zhongshan Golden Bridge Biotechnology, China) and rabbit anti-human caspase-3 (Cell Signaling Technology, Danvers, MA, USA) at $4^{\circ} \mathrm{C}$ overnight. After three washes with PBS, secondary 
antibodies (Maixin Biotechnology Co, Ltd.) were added to the sections and followed by counterstained with hematoxylin and observations under a microscope. All slides were reviewed by pathologists.

\section{Determination of cell viability}

After incubating cells in six different concentrations of the working solutions for $48 \mathrm{~h}$, the media was replaced with $200 \mu \mathrm{l}$ of fresh media and $20 \mu \mathrm{l}$ of MTT $(5 \mathrm{mg} / \mathrm{ml})$. After another $4 \mathrm{~h}, 150 \mu \mathrm{l}$ DMSO was added, followed by shaking the plate for $10 \mathrm{~min}$. The optical density (OD) of each well was measured at 490nm, utilizing a microplate reader. The results were calculated based on the formula: $\mathrm{S}=(\mathrm{OD}$ treated well $-\mathrm{OD}$ blank $) /($ OD control well - OD blank $) \times 100 \%$.

\section{Analysis of apoptosis by flow cytometry}

TFCs apoptosis was measured using flow cytometry (FCM), according to the annexin V-PE/7-AAD kit (BD Bioscience, San Jose, CA, USA) protocol. When the cells had grown to $80 \%$ after $48 \mathrm{~h}$, Nthy-ori3-1 cells were collected and then washed with PBS, resuspended in binding buffer, and incubated with annexin V-PE/7-AAD. We used FlowJo.7.6 software to analyze the FCM results. Approximately $1 \times 10^{4}$ cells were analyzed per each sample. Results were expressed as means \pm SD of three independent experiments.

\section{RNA isolation and reverse transcription-polymerase chain reaction}

\section{(RT-PCR) analysis}

After the cells were treated with KI for 48h, we extracted the total RNA using TRIzol reagent and examined its purity. We then performed RT-PCR to detect mRNA 
expression levels, as previously described [16]. The following primers were synthesized by Beijing Tianyi Huiyuan Biotechnology Co. Ltd. (Beijing, China). human HIF-1 $\alpha$ : forward, ATCCATGTGACCATGAGGAAATG, reverse, TCGGCTAGTTAGGGTACACTTC;

human Caspase-3: forward, CATGGAAGCGAATCAATGGACT, reverse, CTGTACCAGACCGAGATGTCA;

human NDRG1: forward, GTCCTTATCAACGTGAACCCTT, reverse, GCATTGGTCGCTCAATCTCCA;

human GAPDH: forward, GGAGCGAGATCCCTCCAAAAT

reverse, GGCTGTTGTCATACTTCTCATGG.

The results are shown as the mean ratio of the test molecule/GADPH $\pm \mathrm{SD}$.

\section{RNA sequence analysis}

Nthy-ori3-1 cells were treated with different concentrations of KI ( $0 \mathrm{mM}, 40 \mathrm{mM}$, and $80 \mathrm{mM}$ ) for $48 \mathrm{~h}$. Then total RNA was extracted using TRIzol reagent for RNA sequence analysis (Zhejiang Annuoyouda Biotechnology, China). Then the data was analyzed using GSEA.

\section{Statistical analysis}

All the data in this study are presented as mean \pm standard deviation (SD) and were processed using the SigmaPlot 12.5 software. Differences among various groups were analyzed by using one-way ANOVA. All quantitative data were analyzed using SPSS 
version 16.0 (SPSS, Inc., Chicago, USA). P<0.05 was considered statistically significant.

\section{Results}

\section{Increased apoptosis occurs in thyroid tissues from HT patients}

To assess TFC status from HT patients, we detected the expression of caspase- 3 by immunohistochemistry, which is an apoptosis-related protein. The results showed that caspase-3 expression increased in thyroid tissues from HT patients $(n=10)$ when compared to the control group $(\mathrm{n}=10 ; \mathrm{P}<0.05$; Fig. 1 and Table 1$)$. Thus, indicating that apoptosis is elevated in thyroid tissues from HT patients.

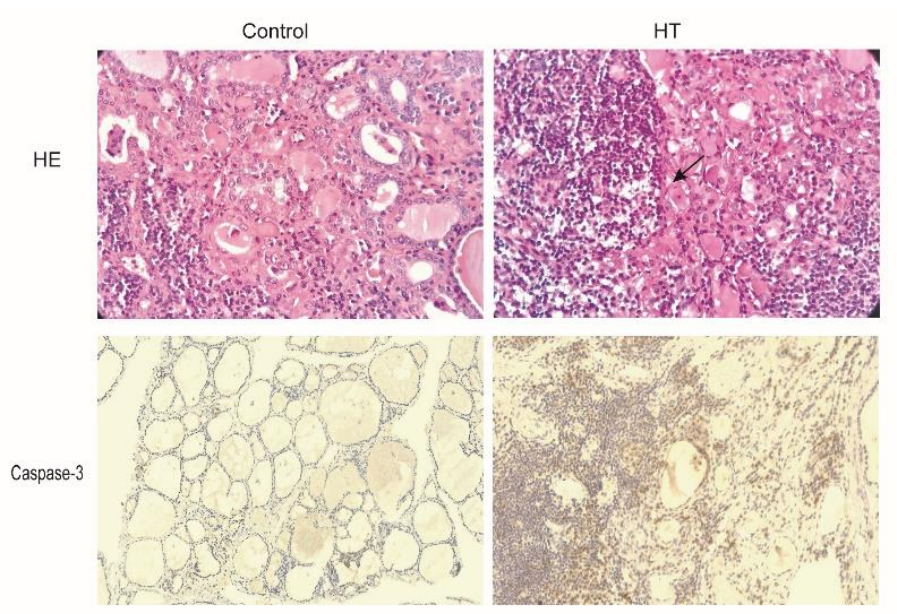

Fig. 1. Representative image of hematoxylin-eosin (HE) staining and caspase-3

immunohistochemical staining in thyroid tissue from HT $(n=10)$ and control $(n=10)$

groups. The black arrow in the HE panel marks acidophilic degeneration in HT tissue.

Brown regions represent caspase-3 positive expression (original magnification, 100×). 
Table 1. Caspase-3 expression in Hashimoto thyroiditis (HT) and control tissues

\begin{tabular}{cccc}
\hline & $\mathrm{HT}$ & Control & P-value \\
& $\mathrm{n}(\%)$ & $\mathrm{n}(\%)$ & \\
\hline Caspase-3 & & & \\
positive & $5(50 \%)$ & $0(0 \%)$ & \\
negative & $5(50 \%)$ & $5(100 \%)$ & \\
\hline
\end{tabular}

$\mathrm{p}<0.05$, HT group vs control group in terms of the expression of caspase- 3

\section{Iodine suppresses proliferation of TFCs in a dose-dependent manner}

Iodine caused concentration-dependent cell injury in the Nthy-ori3-1 thyroid cell line (Fig. 2). After Nthy-ori-3 cells were treated with different concentrations of KI for $24 \mathrm{~h}$, we measured cell viability of all groups by MTT assay. We found that the cell viability in the $40 \mathrm{mM}, 60 \mathrm{mM}$, and $80 \mathrm{mM}$ KI-treated groups were remarkably lower than that of the control group $(\mathrm{P}<0.05)$. Moreover, the cell viability in the group treated with $80 \mathrm{mM}$ KI was significantly lower than the group treated with $40 \mathrm{mM} \mathrm{KI}(\mathrm{P}<0.01)$.

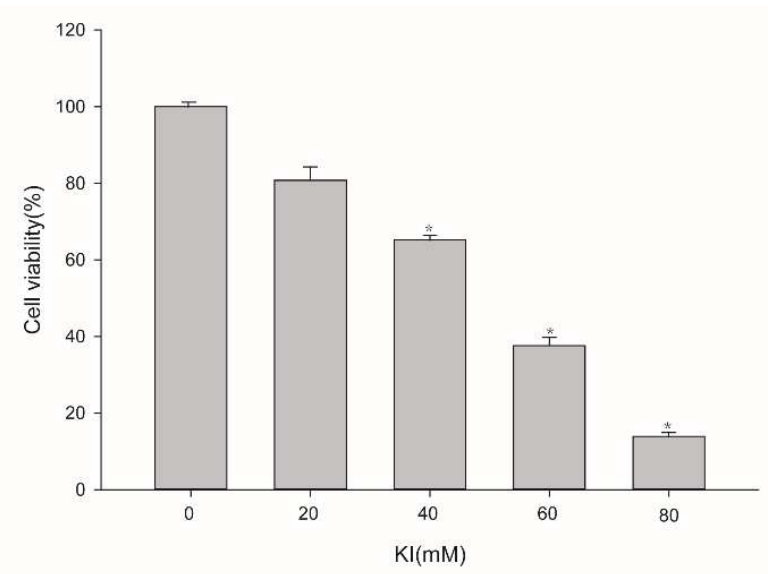

Fig. 2. The effect of iodine in Nthy-ori3-1 thyroid cell line viability. MTT assay results of TFCs treated for $48 \mathrm{~h}$ with different KI concentrations, indicating that iodine caused 
a concentration-dependent decrease in the cell viability of Nthy-ori3-1 thyroid cell line. The bar graphs represent the means \pm SD from 3 independent experiments. ${ }^{*} \mathrm{P}<0.05$ compared to the control group.

\section{Excessive iodine promotes TFCs apoptosis}

Apoptosis of TFCs was measured in Nthy-ori3-1 cells incubated with different KI concentrations for $48 \mathrm{~h}$ by annexin V-PE/7-AAD staining. We found that apoptosis rates in KI-treated TFCs increased markedly in a dose-dependent manner (Fig. 3a). Simultaneously, caspase-3 mRNA expression increased in KI-treated groups when compared to the control group. The $80 \mathrm{mM}$ KI-treated group also showed substantial TFCs apoptosis than other groups $(\mathrm{P}<0.05$, Fig. $3 b)$, suggesting that excessive iodine absorption promotes TFCs apoptosis.
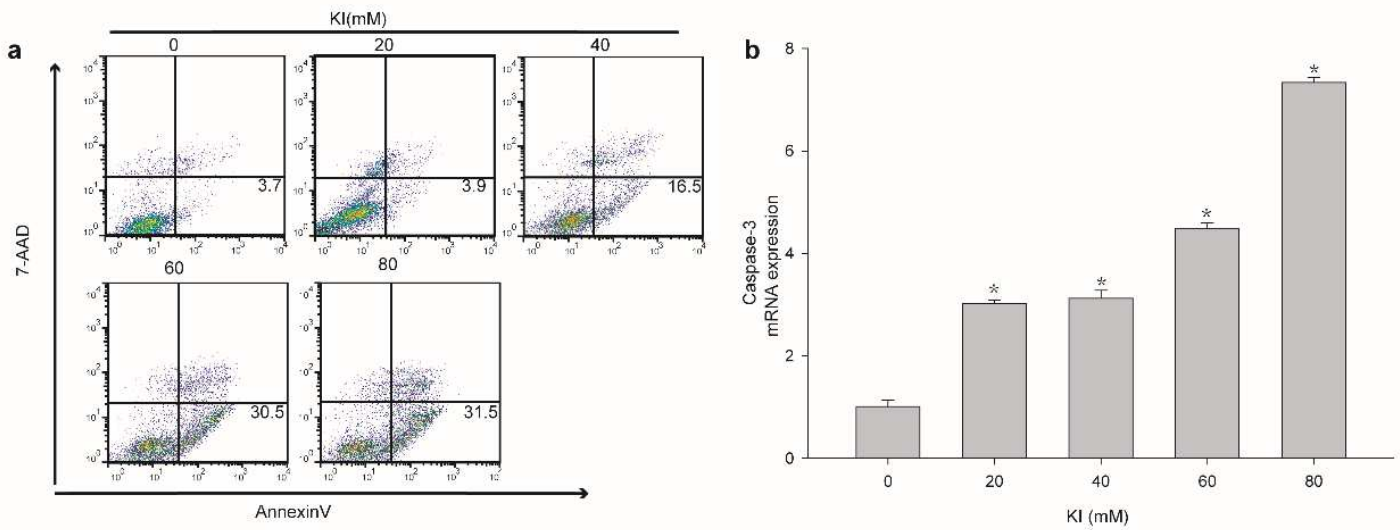

Fig. 3. The effect of iodine in Nthy-ori3-1 thyroid cell line apoptosis. a After thyroid follicular epithelial cells were treated with different concentrations of KI for 48h, TFCs apoptosis was measured by flow cytometry. Apoptosis rates of KI-treated TFCs increased significantly in a dose-dependent manner. b caspase-3 mRNA expression increased in the KI-treated groups compared to the control group. The bar graphs 
represent the means \pm SD from 3 independent experiments. $* \mathrm{P}<0.05$ compared to the control group.

Excessive iodine activates HIF-1 $\alpha$-mediated hypoxia pathway in Nthy-ori-3 cells coincide with upregulation of NDRG1 expression

To further explore the molecular mechanism underlying KI-induced cell apoptosis, we performed RNA sequence analysis of Nthy-ori3-1 cells treated for $48 \mathrm{~h}$ with different KI concentrations $(0 \mathrm{mM}, 40 \mathrm{mM}$, and $80 \mathrm{mM})$. The RNA sequence data revealed that in KI groups treated at a high concentration, the hypoxia pathway is significantly activated (Fig. 4a) and accompanied by upregulation of NDRG1 expression (Fig. 4c, P<0.05, KI treated-groups vs. control group; $\mathrm{P}<0.01,80 \mathrm{mM}$ KI-treated group vs. $40 \mathrm{mM}$ KI-treated group). We then used RT-PCR to detect HIF-1 $\alpha$ and NDRG1 mRNA expression in Nthy-ori-3 cells treated for $48 \mathrm{~h}$ with different KI concentrations. The results showed that HIF-1 $\alpha$ mRNA expression was significantly increased when cells were treated with $\mathrm{KI}$ of $40 \mathrm{mM}$ compared with 0 and $20 \mathrm{mM}$, but decreased when the concentration was above 40mM (Fig. 4b, P<0.05, KI treated-groups vs. control group). NDRG1 mRNA expression levels were positively correlated with KI concentration (Fig. 4d, P<0.05, KI treated-groups vs. control group; $\mathrm{P}<0.01,80 \mathrm{mM}$ KI-treated group vs. $40 \mathrm{mM}$ KI-treated group). Overall, these results suggest that excessive iodine intake could activate the HIF-1 $\alpha$-mediated hypoxia signaling pathway and upregulate NDRG1 expression, promoting TFCs apoptosis. 

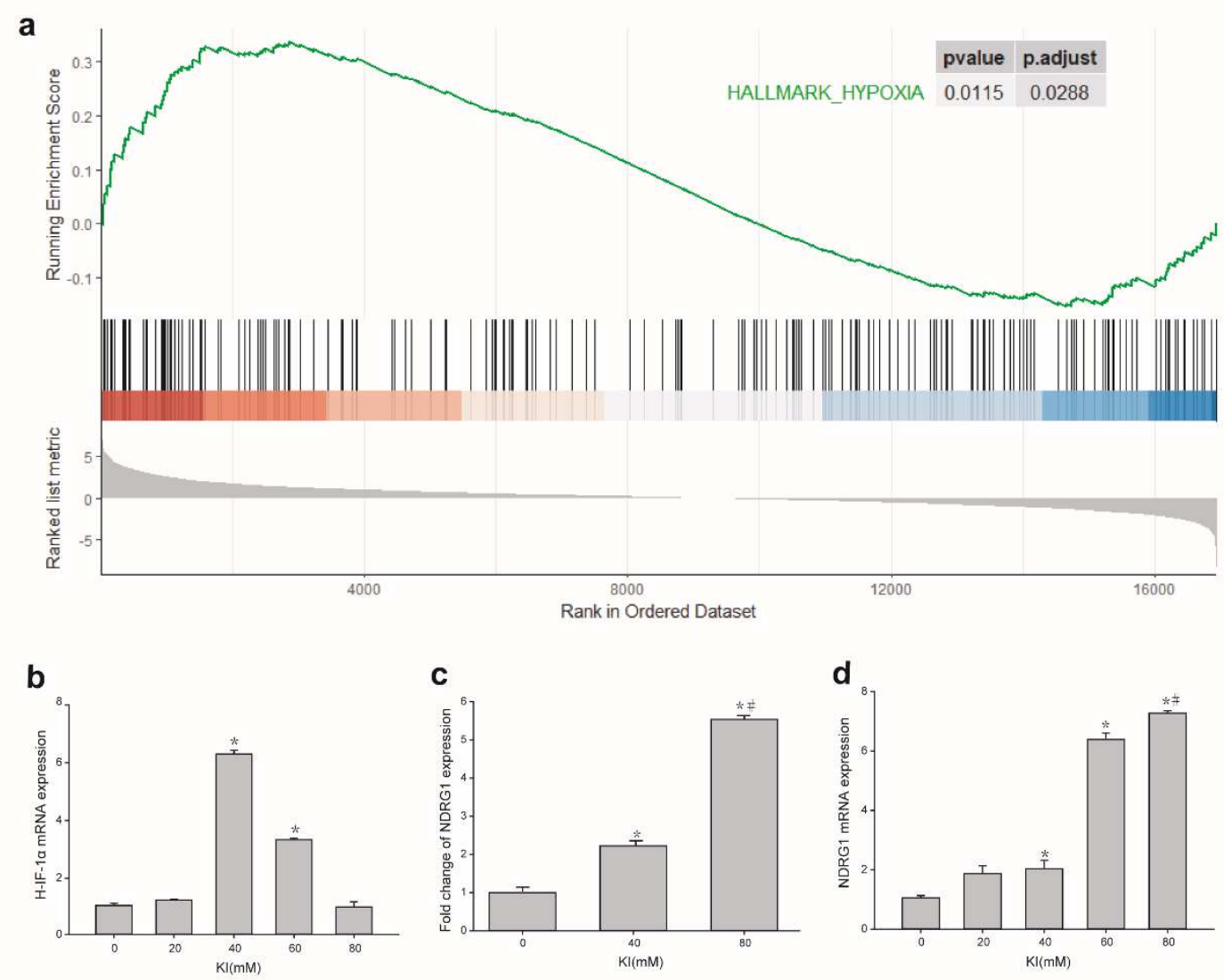

Fig. 4. HIF-1 $\alpha$ mediated hypoxia pathway was activated by excessive iodine stimulation. a RNA sequence analysis suggested that the hypoxia pathway was significantly activated after the cells were exposed for $48 \mathrm{~h}$ to different $\mathrm{KI}$ concentrations $(0 \mathrm{mM}, 40 \mathrm{mM}$, and $80 \mathrm{mM})$. b RT-PCR results showed that HIF-1 $\alpha$ mRNA expression increased when the concentrations of KI was 40mM, but decreased when the concentrations was over 40mM. $\mathbf{c}$ RNA sequence results showed that NDRG1 expression upregulation was associated with hypoxia pathway activation. d RT-PCR results showed that NDRG1 mRNA expression increased along with the increased KI concentrations. The bar graphs represent the mean \pm SD from 3 independent experiments. ${ }^{*} \mathrm{P}<0.05$ compared to the control group; \# $\mathrm{P}<0.01$ compared the $80 \mathrm{mM}$ KI-treated group to the 40mM KI-treated group. 


\section{Discussion}

HT is an organ-specific autoimmune disease that has a genetic predisposition. Despite being an essential microelement for thyroid hormone synthesis, iodine also can contribute directly to the development of thyroid disorders, such as thyroid autoimmunity [17]. To our knowledge, the present study is the first to demonstrate that excessive iodine intake promotes TFCs apoptosis, possibly by activating HIF-1 $\alpha$ mediated hypoxia signaling pathways and upregulating NDRG1 expression.

Apoptosis plays a vital role in HT development. For instance, M. Erdogan found that the atrophy and destruction of thyroid follicles were connected with Fas-mediated apoptosis in HT patients [18]. Fas combined with Fas ligand (FasL) activates caspase8 induced cysteine proteases caspase-3 complex, which selectively affects some proteins activity and leads to TFCs apoptosis. Moreover, activated caspases- 8 initiates the intrinsic apoptotic pathway by Bcl-2 [8]. Therefore, caspase-3 is involved in Fasmediated apoptosis as a key protease. In our study, we found that caspase-3 expression in HT patients was upregulated compared with that of controls. This result agrees with recent reports demonstrating high caspase-3 levels in HT patients [9]. While investigating the effects of excessive iodine on TFCs apoptosis in vitro, we observed that iodine suppressed TFCs proliferation in a dose-dependent manner and promoted the expression of apoptosis-related proteins. Similarly, the expression of caspase-3 in HT tissue also increased, indicating that excessive iodine may promote HT development. 
Nevertheless, the results of this study suggest that iodine could induce HT development by activating the apoptosis signaling pathway in TFCs, the molecular mechanism behind this process was unclear. Furthermore, there was evident activation of the hypoxia signaling pathway under high KI concentrations. PCR analysis from in vitro experiments corroborated that the expression of HIF- $1 \alpha$ and caspase- 3 in iodinetreated Nthy-ori3-1 cells increased along with increased concentrations. Therefore, we speculate that excessive iodine may induce TFCs apoptosis through the activation of the HIF-1 $\alpha$-mediated pathway.

Under hypoxic conditions, HIF-1 $\alpha$ could bind to HIF-1 $\beta$ to form the HIF-1 complex. Consequently, this complex formation could activate gene expression by binding to hypoxia response elements (HREs) located in the promoter region of some genes such as NDRG1 $[19,20,21]$. The NDRG1 gene is a member of the N-myc downregulated genes and encodes the NDRG1 protein. This protein consists of 394 amino acids and is widely expressed in the cytoplasm of different cell types. NRDG1 is mainly involved in apoptosis, proliferation, migration, and angiogenesis processes [22]. A recent study showed that high NDRG1 expression levels resulted from the activation of the HIF-1 signal during hypoxia [23]. On the other hand, there is strong evidence showing that the NDRG1 gene possessed two HREs located at -1376 and $-7503 \mathrm{bp}$. Thus, HIF-1 could induce NDRG1 expression by binding to its HREs [24]. Our in vitro PCR analysis showed that the expression of NDRG1 in iodine-treated Nthy-ori3-1 cells is proportionally increased. Taken together, these findings suggest that excessive iodine may activate the HIF-1 $\alpha$-mediated hypoxia signaling pathway and upregulate 
expression of NDRG1, therefore promote apoptosis of TFCs and the development of HT. However, in our in vitro study, HIF- $1 \alpha$ mRNA level raised on the treatment with $40 \mathrm{mM} \mathrm{KI}$ but decreased when the concentration is over $40 \mathrm{mM}$, likely some unknown factors are involved in this pathway.

In conclusion, our study confirmed that excessive iodine adsorption activates the HIF-1 $\alpha$-mediated hypoxia pathway in the TFCs apoptosis process. An increased apoptosis rate was also observed in HT patients. These results suggest that excessive iodine may promote the development of HT by inducing TFCs apoptosis. The present study raises the possibility that HIF-1 $\alpha$ may be a potential biomarker of thyrocyte injury in HT. Therefore, we should take up appropriate iodine to reduce the prevalence of HT.

Our study has some limitations. First, we only confirmed the mRNA expression of related genes; however, the protein expression levels should also be determined. Second, in vitro studies do not fully represent in vivo studies. We will address these limitations in future studies to explain the mechanism underlying HT development.

Funding This study was funded by the Research Foundation of Beijing Friendship Hospital, Capital Medical University (NO. yyqdkt2019-18)

\section{Compliance with ethical standards}

Conflicts of interest The authors declare that they have no conflict of interest

Ethical approval All procedures performed in studies involving human participants were in accordance with the ethical standards of the Ethics Committee of Beijing Friendship Hospital affiliated to Capital Medical University (reference number: 2020-P2-123-01) and with the 1964 helsinki declaration and its later amendments or comparable ethical standards. 
Informed consent Informed consent was obtained from all individual participant included in the study.

\section{References}

1. Zimmermann MB, Boelaert K, Iodine deficiency and thyroid disorders. The Lancet Diabetes \& Endocrinology 3, 286-295 (2015).

2. Taylor PN, Albrecht D, Scholz A, Gutierrez-Buey G, Lazarus JH, Dayan CM, Okosieme OE, Global epidemiology of hyperthyroidism and hypothyroidism. Nature Reviews Endocrinology 14, 301-316. (2018).

3. Teng W, Shan Z, Teng X, Guan H, Li Y, Teng D, Jin Y, Yu X, Fan C, Chong W, Yang F, Dai H, Yu Y, Li J, Chen Y, Zhao D, Shi X, Hu F, Mao J, Gu X, Yang R, Tong Y, Wang W, Gao T, Li C Effect of iodine intake on thyroid diseases in China. The New England journal of medicine 354, 2783-2793 (2006).

4. Pedersen IB, Knudsen N, Carle A, Vejbjerg P, Jorgensen T, Perrild H, Ovesen L, Rasmussen LB, Laurberg P, A cautious iodization programme bringing iodine intake to a low recommended level is associated with an increase in the prevalence of thyroid autoantibodies in the population. Clinical endocrinology 75, 120-126 (2011).

5. Resende de Paiva C, Gronhoj C, Feldt-Rasmussen U, von Buchwald C, Association between Hashimoto's Thyroiditis and Thyroid Cancer in 64,628 Patients. Frontiers in oncology 7, 53 (2017). 6. Antonelli A, Ferrari SM, Corrado A, Di Domenicantonio A, Fallahi P, Autoimmune thyroid disorders. Autoimmunity reviews 14, 174-180 (2015).

7. Hu S, Rayman MP Multiple, Nutritional Factors and the Risk of Hashimoto's Thyroiditis. Thyroid : official journal of the American Thyroid Association 27, 597-610 (2017).

8. Pyzik A, Grywalska E, Matyjaszek-Matuszek B, Roliński J, Immune Disorders in Hashimoto’s Thyroiditis: What Do We Know So Far? Journal of Immunology Research 2015, 1-8 (2015).

9. Xu C, Wu F, Mao C, Wang X, Zheng T, Bu L, Mou X, Zhou Y, Yuan G, Wang S, Xiao Y, Excess iodine promotes apoptosis of thyroid follicular epithelial cells by inducing autophagy suppression and is associated with Hashimoto thyroiditis disease. Journal of Autoimmunity 75, 50-57 (2016).

10. Duntas L, The Role of Iodine and Selenium in Autoimmune Thyroiditis. Hormone and Metabolic Research 47, 721-726 (2015).

11. Wang S, Liu Y, Zhao N, Cui X, Huang M, Li Y, Shan Z, Teng W IL-34 Expression Is Reduced in Hashimoto's Thyroiditis and Associated With Thyrocyte Apoptosis. Frontiers in Endocrinology 9, 1-9 (2018).

12. Sethi K, Rao K, Bolton D, Patel O, Ischia J, Targeting HIF-1 $\alpha$ to Prevent Renal Ischemia-Reperfusion Injury: Does It Work? International Journal of Cell Biology 2018, 1-7 (2018).

13. Delbrel E, Soumare A, Naguez A, Label R, Bernard O, Bruhat A, Fafournoux P, Tremblais G, Marchant D, Gille T, Bernaudin JF, Callard P, Kambouchner M, Martinod E, Valeyre D, Uzunhan Y, Planes C, Boncoeur E, HIF-1 alpha triggers ER stress and CHOP-mediated apoptosis in alveolar epithelial cells, a key event in pulmonary fibrosis. Scientific reports 8, 17939 (2018).

14. Zhao Q, Tan BB, Li Y, Fan LQ, Yang PG, Tian Y, Enhancement of Drug Sensitivity by Knockdown of HIF-1alpha in Gastric Carcinoma Cells. Oncology research 23, 129-136 (2016).

15. Caturegli P, De Remigis A, Rose NR, Hashimoto thyroiditis: Clinical and diagnostic criteria. Autoimmunity reviews 13, 391-397 (2014).

16. Liu H, Zeng Q, Cui Y, Yu L, Zhao L, Hou C, Zhang S, Zhang L, Fu G, Liu Y, Jiang C, Chen X, Wang A, The effects and underlying mechanism of excessive iodide on excessive fluoride-induced thyroid 
cytotoxicity. Environmental toxicology and pharmacology 38, 332-340 (2014).

17. Kolypetri P, King J, Larijani M, Carayanniotis G, Genes and Environment as Predisposing Factors in Autoimmunity: Acceleration of Spontaneous Thyroiditis by Dietary Iodide in NOD.H2h4Mice. International Reviews of Immunology 34, 542-556 (2015).

18. Erdogan M, Kulaksizoglu M, Ganidagli S, Berdeli A, Fas/FasL gene polymorphism in patients with Hashimoto's thyroiditis in Turkish population. Journal of Endocrinological Investigation 40, 77-82 (2016).

19. Choi SH, Kim MY, Yoon YS, Koh DI, Kim MK, Cho SY, Kim KS, Hur MW, Hypoxia-induced RelA/p65 derepresses SLC16A3 (MCT4) by downregulating ZBTB7A. Biochimica et biophysica acta Gene regulatory mechanisms 1862, 771-785 (2019).

20. Zhao T, Jin F, Xiao D, Wang H, Huang C, Wang X, Gao S, Liu J, Yang S, Hao J, IL-37/ STAT3/

HIF-1alpha negative feedback signaling drives gemcitabine resistance in pancreatic cancer. Theranostics 10, 4088-4100 (2020).

21. Said HM, Safari R, Al-Kafaji G, Ernestus R-I, Löhr M, Katzer A, Flentje M, Hagemann C, Time-and oxygen-dependent expression and regulation of NDRG1 in human brain cancer cells. Oncology Reports 37, 3625-3634 (2017).

22. Sahni S, Park KC, Kovacevic Z, Richardson DR, Two mechanisms involving the autophagic and proteasomal pathways process the metastasis suppressor protein, N-myc downstream regulated gene 1 . Biochimica et Biophysica Acta (BBA) - Molecular Basis of Disease 1865, 1361-1378 (2019).

23. Guo D-D, Xie K-F, Luo X-J, Hypoxia-induced elevated NDRG1 mediates apoptosis through reprograming mitochondrial fission in HCC. Gene 741, 144552 (2020).

24. Park KC, Paluncic J, Kovacevic Z, Richardson DR, Pharmacological targeting and the diverse functions of the metastasis suppressor, NDRG1, in cancer. Free radical biology \& medicine 157,154-175 (2020). 


\section{Figures}

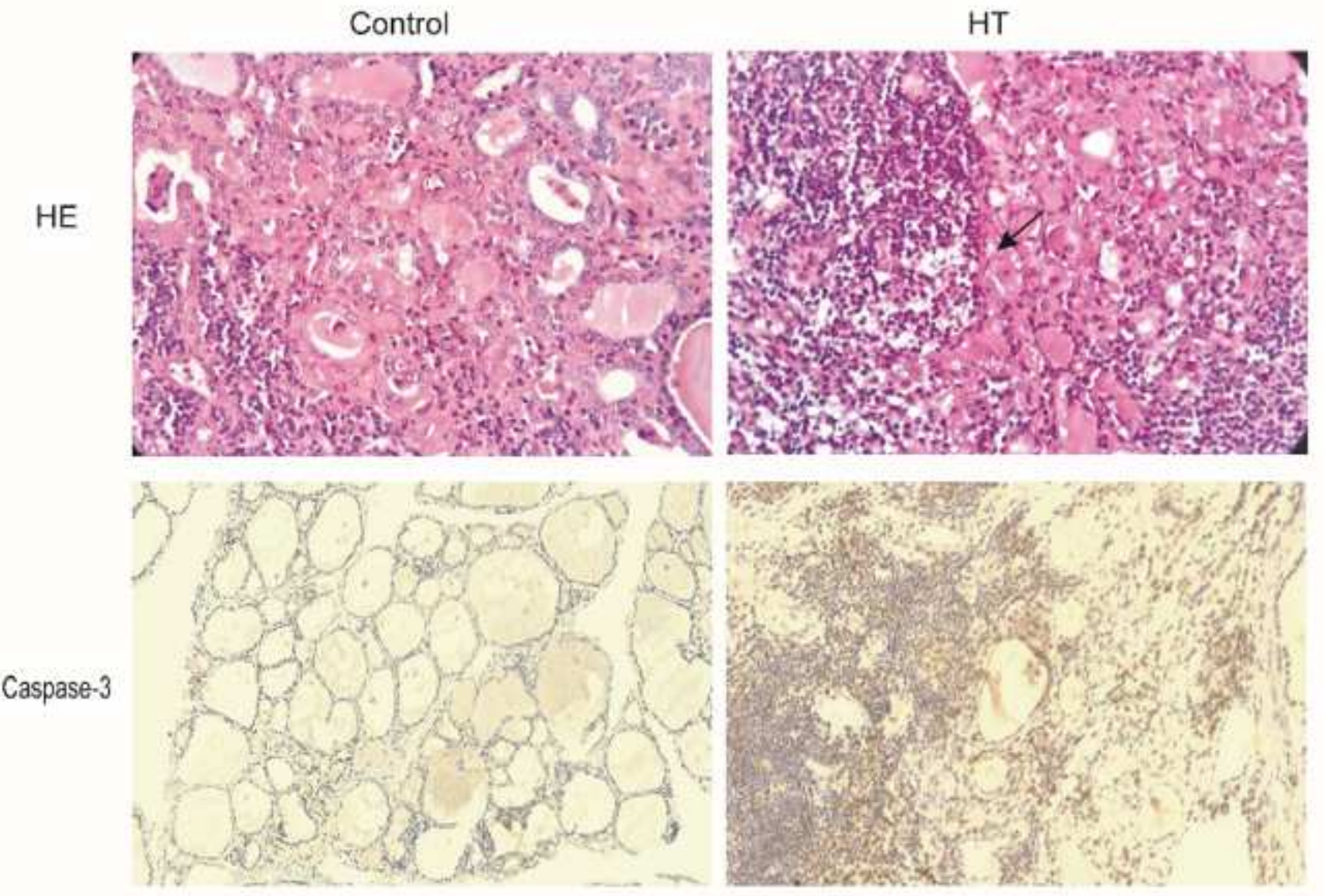

\section{Figure 1}

Representative image of hematoxylin-eosin (HE) staining and caspase-3 immunohistochemical staining in thyroid tissue from HT $(n=10)$ and control $(n=10)$ groups. The black arrow in the HE panel marks acidophilic degeneration in $\mathrm{HT}$ tissue. Brown regions represent caspase-3 positive expression (original magnification, 100x). 


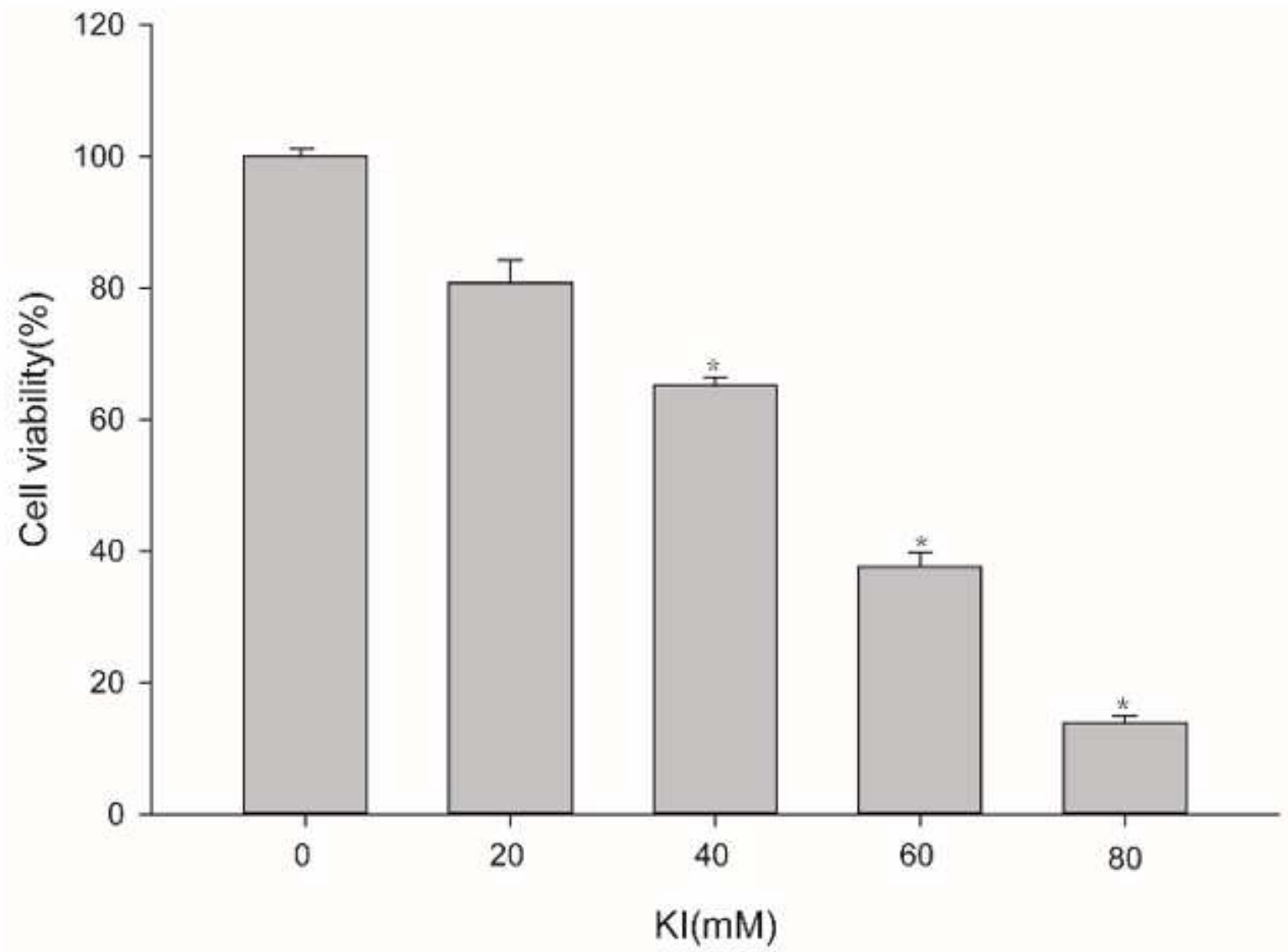

Figure 2

The effect of iodine in Nthy-ori3-1 thyroid cell line viability. MTT assay results of TFCs treated for $48 \mathrm{~h}$ with different $\mathrm{KI}$ concentrations, indicating that iodine caused a concentration-dependent decrease in the cell viability of Nthy-ori3-1 thyroid cell line. The bar graphs represent the means \pm SD from 3 independent experiments. ${ }^{*} \mathrm{P}<0.05$ compared to the control group.

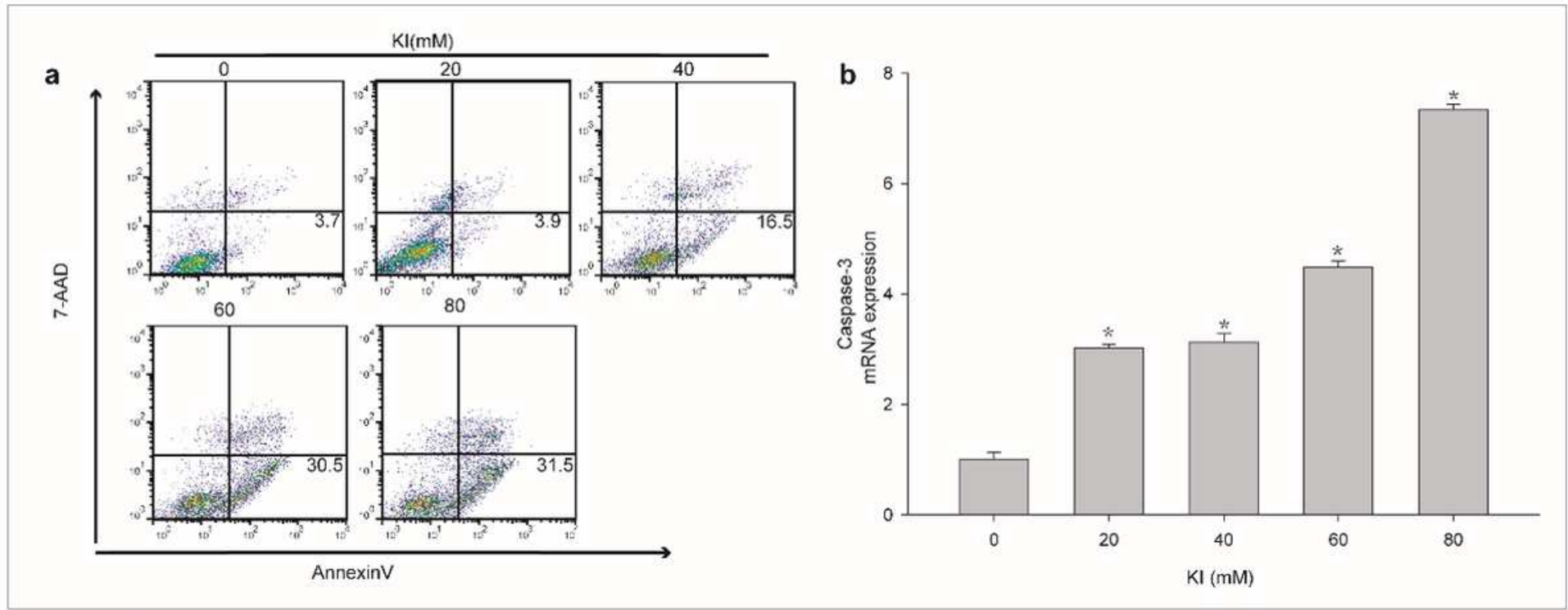




\section{Figure 3}

The effect of iodine in Nthy-ori3-1 thyroid cell line apoptosis. a After thyroid follicular epithelial cells were treated with different concentrations of KI for 48h, TFCs apoptosis was measured by flow cytometry. Apoptosis rates of KI-treated TFCs increased significantly in a dose-dependent manner. b caspase-3 mRNA expression increased in the KI-treated groups compared to the control group. The bar graphs represent the means $\pm S D$ from 3 independent experiments. ${ }^{*} P<0.05$ compared to the control group.
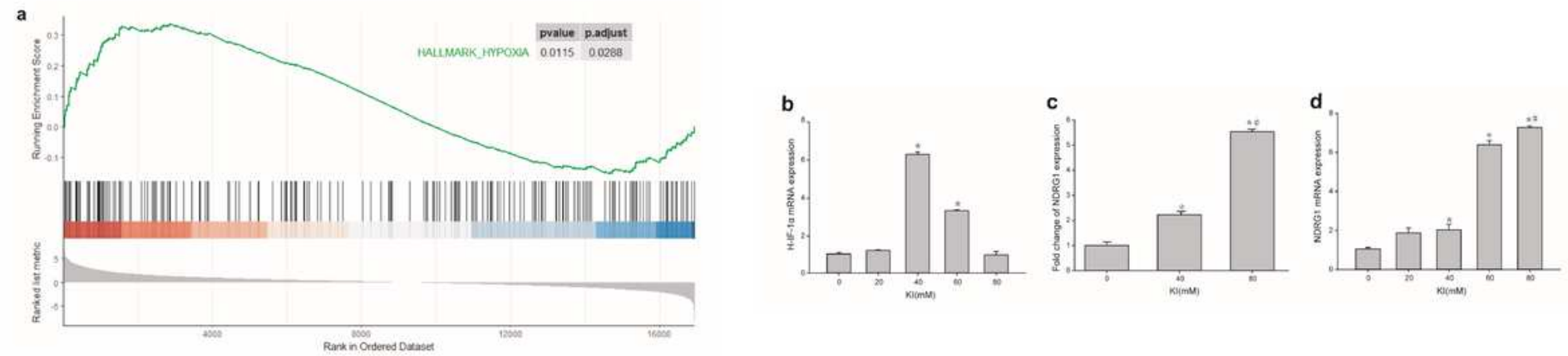

Figure 4

HIF-1a mediated hypoxia pathway was activated by excessive iodine stimulation. a RNA sequence analysis suggested that the hypoxia pathway was significantly activated after the cells were exposed for $48 \mathrm{~h}$ to different $\mathrm{KI}$ concentrations $(0 \mathrm{mM}, 40 \mathrm{mM}$, and $80 \mathrm{mM})$. b RT-PCR results showed that HIF-1a mRNA expression increased when the concentrations of $\mathrm{KI}$ was $40 \mathrm{mM}$, but decreased when the concentrations was over 40mM. c RNA sequence results showed that NDRG1 expression upregulation was associated with hypoxia pathway activation. $d$ RT-PCR results showed that NDRG1 mRNA expression increased along with the increased $\mathrm{KI}$ concentrations. The bar graphs represent the mean $\pm S D$ from 3 independent experiments. ${ }^{*} \mathrm{P}<0.05$ compared to the control group; \# $\mathrm{P}<0.01$ compared the $80 \mathrm{mM} \mathrm{Kl}$-treated group to the $40 \mathrm{mM} \mathrm{KI}$-treated group. 